\title{
Cerebral Salt-Wasting Syndrome in Subarachnoid Hemorrhage: A Primer for Interventional Neuroradiologists
}

\author{
Adhithyan Rajendran ${ }^{1}$ Santhosh Kumar Kannath ${ }^{2}$ \\ ${ }^{1}$ Department of Imaging Sciences and Interventional Radiology, \\ Sree Chitra Tirunal Institute of Medical Sciences and Technology, \\ Trivandrum, Kerala, India \\ ${ }^{2}$ Neurointervention Center, Department of Imaging Sciences and \\ Interventional Radiology, Sree Chitra Tirunal Institute of Medical \\ Sciences and Technology, Trivandrum, Kerala, India \\ ${ }^{3}$ Department of Neuro-anaesthesia, Sree Chitra Tirunal Institute of \\ Medical Sciences and Technology, Trivandrum, Kerala, India \\ J Clin Interv Radiol ISVIR 2018;2:51-54
}

\author{
Smitha Vimala ${ }^{3}$ Jayadevan Enakshy Rajan²
}

\begin{abstract}
Keywords

- basilar top aneurysm

- electrolyte imbalance

- salt wasting
\end{abstract}

\begin{abstract}
Address for correspondence Dr. Santhosh Kumar Kannath, MD, PDCC, Associate Professor, Department of Imaging Sciences and Interventional Radiology, Neurointervention Center, Sree Chitra Tirunal Institute of Medical Sciences and Technology, Trivandrum, Kerala 695011, India (e-mail: drsanthoshkannath@gmail.com).
\end{abstract}

\section{Introduction}

Hyponatremia is a commonly seen in patients with neurosurgical diseases. When hyponatremia is associated with central nervous system disease, first possibility one would normally consider is syndrome of inappropriate antidiuretic hormone secretion (SIADH). Another uncommonly diagnosed but a frequent cause of severe hyponatremia in postneurosurgical procedures is cerebral salt-wasting syndrome (CSWS). ${ }^{1}$ Treatments are entirely different for these two conditions, as vigorous sodium and fluid replacement is the treatment of choice for CSWS, whereas fluid restriction is the treatment of choice in SIADH. Therefore, there is a need to differentiate these two conditions on an emergency basis, and management must be done appropriate to the condition. CSWS, specifically in neurosurgical cases, has been reported sporadically in neurosurgery and neurology journals. The identification of CSWS is important for the treating interventional radiologist/neuroradiologist, as they are increasingly involved in the management of various neurovascular diseases. The clinical recognition and management of CSWS are highlighted in the following prototypic case.

\section{Case Description}

\section{Brief Clinical History}

A 64-year-old diabetic and hypertensive woman presented with sudden-onset severe intensity holocranial headache and multiple episodes of vomiting. She was evaluated in a nearby hospital with plain computed tomography (CT), followed by CT angiography, which showed a basilar top aneurysm and subarachnoid hemorrhage (SAH, fisher grade 3 ). She was referred to neurointerventional department of the hospital for further management. On admission patient was stable, her Glasgow coma scale (GCS) was $15 / 15$, with no neurologic deficits. Cerebral angiogram ( - Fig. 1A) was done on the fifth post ictus day, and it showed a wide necked basilar top aneurysm, measuring $12 \times 11 \times 10 \mathrm{~mm}$, incorporating
Dol https://doi.org/

10.1055/s-0037-1609047.

ISSN 2457-0214.
Copyright $@ 2018$ by Indian Society

of Vascular and Interventional

Radiology
License terms

()(1) $\ominus \circledast$ 

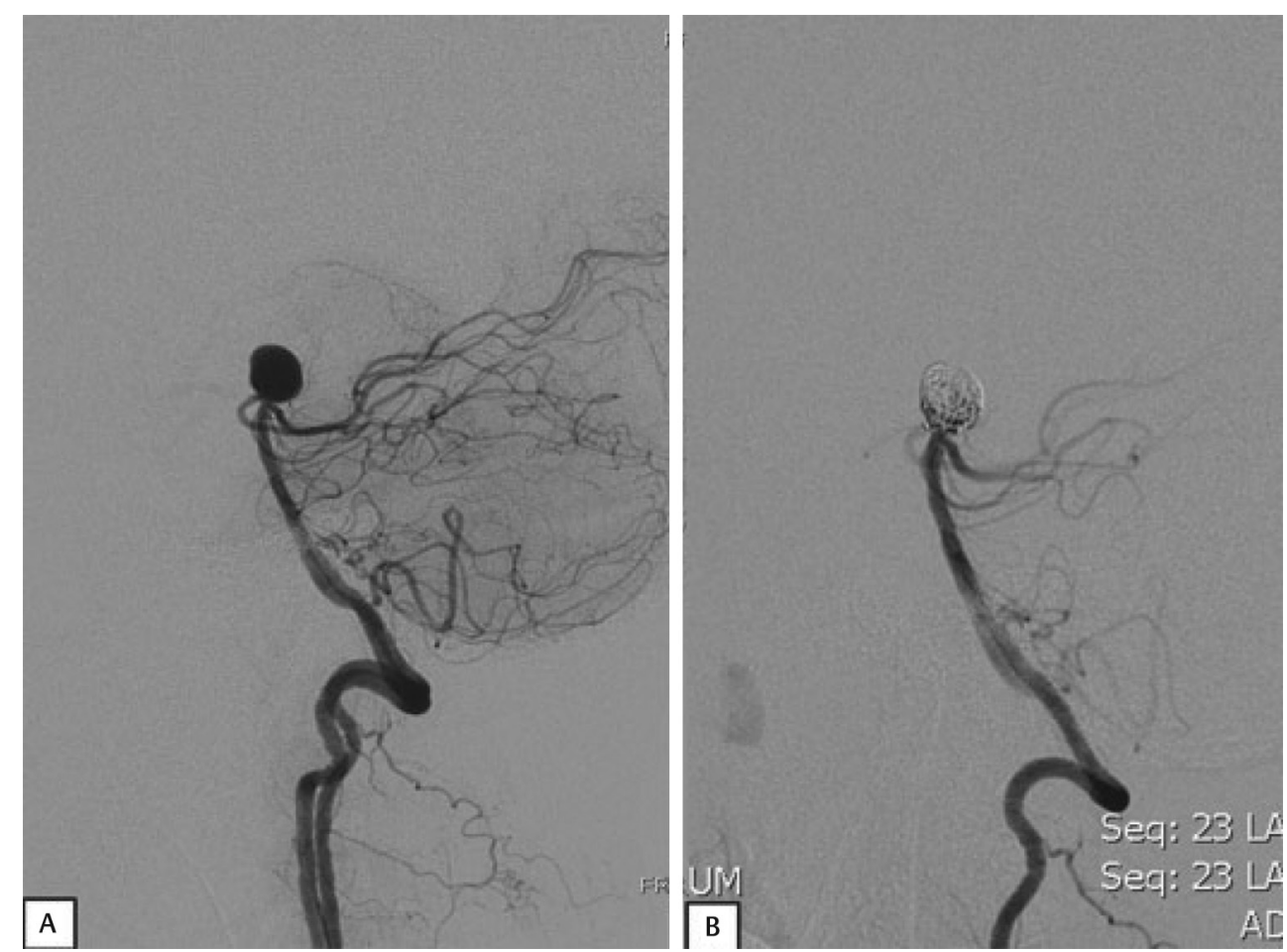

Fig. 1 DSA lateral projection (A) showing a wide-necked basilar top aneurysm, DSA post near-complete coiling (B) lateral projection image.

both posterior cerebral arteries (PCA). Endovascular coiling of the aneurysm ( - Fig. 1B) with balloon assistance was done under general anesthesia on the eighth post ictus day. The patient was extubated on the same day after procedure, and she was stable with no neurologic deficits. She received oral nimodipine, phenytoin, and dexamethasone from outside institution, which was continued during hospitalization.

\section{Clinical Course during Hospitalization}

In the immediate postoperative day (eighth day of ictus), her serum potassium level was found low $(2.6 \mathrm{mEq} / \mathrm{dL})$, which was initially corrected with injection of potassium chloride $(\mathrm{KCl})$ of $40 \mathrm{mEq}$ and later with oral $\mathrm{KCl}$ supplements. She became intermittently drowsy on the ninth day of ictus, and magnetic resonance imaging (MRI) including diffusion sequence showed no acute infarcts or other additional observations. Her serum sodium levels dropped to $124 \mathrm{mEq} /$ $\mathrm{dL}$ from baseline value of $135 \mathrm{mEq} / \mathrm{dL}$. Oral salt correction was initially attempted, and her sodium level remains static with no further drop for the next 4 days. Her hemoglobin was low in postinterventional period $(8.5 \mathrm{~g} \%)$, which was corrected with 1 unit of packed red blood cell. Her hemoglobin, however, rose to $12.4 \mathrm{~g} \%$, which was more than the expected correction with a single unit of transfusion, and this observation suggested the existence of negative water balance. On fifth post coiling day, her drowsiness worsened and clinical examination revealed moderate hydration and postural hypotension.

Serum and urine biochemistry was done for further evaluation. Her serum sodium dropped further to $112 \mathrm{mEq} / \mathrm{L}$, but her serum potassium was within normal limits $(4 \mathrm{mEq} / \mathrm{L})$. Urine-specific gravity was 1.016 . Serum osmolality decreased to $268 \mathrm{mOsm} / \mathrm{L}$ and urine osmolality was $216 \mathrm{mOsm} / \mathrm{L}$. Her urinary output was $3,800 \mathrm{~mL}$ with a negative water imbalance of $340 \mathrm{~mL}$. Intravenous correction of serum sodium level was initiated with $200 \mathrm{~mL}$ of intravenous 3\% sodium over 8 hours, followed by maintenance intravenous normal saline. Next day, her drowsiness improved and her serum sodium increased above $120 \mathrm{mEq} / \mathrm{L}$. Aggressive oral and intravenous sodium correction was continued for the next 3 days, and her serum sodium levels stabilized above $120 \mathrm{mEq} / \mathrm{L}$. At discharge, her serum sodium was $123 \mathrm{mEq} / \mathrm{L}$ and the patient remained fully conscious, ambulant with no significant complaints. Her serum sodium level at 3-month clinical follow-up was $135 \mathrm{mEq} / \mathrm{L}$.

\section{Analysis of Clinical History and Biochemical Investigations}

Hyponatremia is defined as a serum sodium level of less than $135 \mathrm{mEq} / \mathrm{L}$. Thorough history and physical examination, followed by laboratory investigations, can guide the physician to establish the possible cause of hyponatremia in SAH patients.

On clinical assessment, patients with CSWS will have features of hypovolemia, such as orthostatic changes in blood pressure and pulse, dry mucous membranes, flat neck veins, weight loss, and negative fluid balance. In SIADH, the patient will be either normovolemic or euvolemic, with positive water balance. The patient under discussion had negative water balance, orthostatic hypotension, giddiness, and dry mouth, all of which favors CSWS.

First step in biochemical investigations is to evaluate the serum sodium level to assess the degree of hyponatremia and categorize into mild (130-135 mEq/L), moderate (125-130 $\mathrm{mEq} / \mathrm{L})$, or profound $(<125 \mathrm{mEq} / \mathrm{L})$. The patient had initially moderate hyponatremia followed by profound hyponatremia. 
The next step is to evaluate whether the serum osmolality is low, normal (275-295 mOsm/L), or increased. When it is normal or increased, the differentials include hyperlipidemia, hyperproteinemia, hyperglycemia, or mannitol infusions. The serum osmolality in CSWS or SIADH will be less. The serum osmolality in this patient measured approximately $268 \mathrm{mOsm} / \mathrm{L}$, which excluded the cause of hyponatremia due to endogenous macromolecules

Then urine osmolality must be then evaluated. The normal random urine osmolality should average between 300 and $900 \mathrm{mOsm} / \mathrm{L}$. Some cases of hypotonic hyponatremia may be due to primary polydipsia, and in such patients, urine osmolality will be less than $100 \mathrm{mOsm} / \mathrm{L}$ due to preserved kidney dilution capacity. The urine osmolality of CSWS and SIADH is usually high. In this patient, urine osmolality was $216 \mathrm{mOsm} / \mathrm{L}$.

The next step is to assess the random urinary sodium concentration of the patient. Urinary sodium will be low $(<20 \mathrm{mEq} / \mathrm{L})$ in hypovolemia cases, whereas it will be elevated $(>40 \mathrm{mEq} / \mathrm{L})$ in SIADH as well as in CSWS. In this case, the patient had high urine sodium level of $51 \mathrm{mOsm} / \mathrm{L}$.

Assessment of water balance helps in the differentiation of CSWS from SIADH. The output is unusually high in CSWS with negative fluid balance. This patient had high urinary output with negative water balance suggesting a clinical diagnosis of CSWS. Other investigations such as serum creatinine, blood urea nitrogen, hematocrit (as in this case), fractional excretion of uric acid, phosphate, and urate after correction may help identify hemoconcentration that would favor the diagnosis of CSWS. - Table 1 highlights the important clinical and biochemical parameters to differentiate CSWS and SIADH.

\section{Management of Cerebral Salt-Wasting Syndrome}

In symptomatic patients with acute hyponatremia (duration of $<48$ hours), urgent correction by 4 to $6 \mathrm{mmol} / \mathrm{L}$ has to be initiated to prevent brain herniation and neurologic damage from cerebral ischemia. ${ }^{2}$ For severe symptoms, intravenous infusion of 100 to $150 \mathrm{~mL}(2 \mathrm{~mL} / \mathrm{kg})$ of $3 \% \mathrm{NaCl}$ over 15 to 20 minutes could be administered, repeated if necessary, to attain an increase in serum sodium values up to $2 \mathrm{mmol} / \mathrm{L}$ in the first hour and up to 4 to $5 \mathrm{mmol} / \mathrm{L}$ in 4 hours. This rapid correction is always with the objective to control the severe symptoms, mainly seizures. In patients with mild to moderate symptoms, $3 \% \mathrm{NaCl}$ is infused at 0.5 to $1 \mathrm{~mL} / \mathrm{kg} / \mathrm{h}$.

This patient was finally diagnosed as CSWS and hyponatremia correction along with fluid resuscitation was

Table 1 Overlapping findings and clinical, biochemical differences between CSWS and SIADH

\begin{tabular}{|c|c|c|}
\hline Parameters & CSWS & SIADH \\
\hline Serum sodium & Low & Low \\
\hline Urine sodium & Increased (> $40 \mathrm{mEq} / \mathrm{L}$ ) & Increased (> $40 \mathrm{mEq} / \mathrm{L}$ ) \\
\hline Serum osmolality & Decreased & Decreased \\
\hline Urine osmolality & High & High \\
\hline Urine volume & Increased & Low or normal \\
\hline Water balance & Negative & Normal/positive \\
\hline Sodium balance & Negative & Variable \\
\hline Extracellular volume & Low & Normal/increased \\
\hline Hematocrit & Increased & Normal \\
\hline Serum creatinine & Increased & Decreased \\
\hline Blood urea nitrogen & Increased & Decreased \\
\hline Serum potassium & Normal/increased & Normal \\
\hline Serum uric acid & Normal/decreased & Decreased $(<4 \mathrm{mg} / \mathrm{dL})$ \\
\hline FEUA $^{a}$ & Increased & Increased \\
\hline After correction of hyponatremia & $\begin{array}{l}\text { Serum uric acid and increased FENa } \\
\text { persist }\end{array}$ & $\begin{array}{l}\text { Serum uric acid and increased FENa } \\
\text { resolve }\end{array}$ \\
\hline FEP & Elevated & Normal \\
\hline Patient condition & $\begin{array}{l}\text { Postural hypotension, dry mucous } \\
\text { membranes. }\end{array}$ & No signs of dehydration \\
\hline Central venous pressure & Low & Increased or normal \\
\hline Pulmonary capillary wedge pressure & Low & Increased or normal \\
\hline Brain natriuretic peptide & Increased & Normal \\
\hline Management & $\begin{array}{l}\text { Fluid and sodium replacement, } \\
\text { Fludrocortisone }\end{array}$ & Water restriction, Demeclocycline \\
\hline
\end{tabular}

Abbreviations: CSWS, cerebral salt-wasting syndrome; FENa, fractional excretion of Na; FEP, fractional excretion of phosphate; FEUA, fractional excretion of uric acid; SIADH, syndrome of inappropriate antidiuretic hormone secretion.

aFEUA $=($ Urinary uric acid $[\mathrm{mg} / \mathrm{mL}] \times$ serum creatinine $[\mathrm{mg} / \mathrm{mL}] \div($ serum uric acid $[\mathrm{mg} / \mathrm{mL}] \times$ urinary creatinine $[\mathrm{mg} / \mathrm{mL}]) \times 100 \%$. Normal $=<10 \%$. 
started. Over 8 hours, $200 \mathrm{~mL}$ of $3 \%$ sodium infusion was administered to raise her sodium level by $4 \mathrm{mmol} / \mathrm{L}$ (rapid correction) along with infusion of normal saline for correction of hypovolemia. After this, normal saline infusion was continued for slow correction for the first 24 hours and oral salt was given to correct the serum sodium, once serum sodium was elevated above $120 \mathrm{mEq} / \mathrm{L}$. These measures were repeated over several days to maintain her sodium in the range of 123 to $127 \mathrm{mEq} / \mathrm{L}$. Because CSWS may persist for 3 to 4 weeks after the insulting event, the patient was on close monitoring of serum electrolytes after discharge.

\section{Discussion}

Hyponatremia is commonly seen following SAH and is frequently attributed to either CSWS or SIADH. Cerebral salt wasting is a disorder of sodium and water transport in the kidneys that occurs in patients with cerebral diseases in the setting of normal adrenal and thyroid function, leading to hyponatremia and extracellular volume depletion. Two mechanisms have been discussed in literature regarding mechanism of CSWS. One is increased activity of the sympathetic nervous system and dopamine release due to brain injury, leading to elevated renal pressure-natriuresis response and urinary sodium loss. Another mechanism is probably due to the release of brain natriuretic peptides by the injured brain.

In a retrospective study by Kao et al, ${ }^{3}$ hyponatremia was detected in $59.2 \%$ patients, of whom $35.4 \%$ were categorized as SIADH and $22.9 \%$ as CSWS. They also found that hyponatremia was associated with significantly longer hospital stay (17 days in hyponatremic group vs. 9 days in eunatremic patients group) compared with eunatremic patients. Therefore, there is a need to assess the hyponatremia, its cause, and manage it appropriately. In a retrospective study of 259 patients of SAH, it was shown that advanced age and smoking were associated with increased likelihood and duration of hyponatremia respectively. ${ }^{4}$ Therefore, older patients and patients with a smoking history should therefore be monitored more closely for hyponatremia and may benefit from more aggressive therapy.

Both CSWS and SIADH present with low serum osmolality, high urine osmolality, and a high urine sodium level. Basic difference between these two conditions is the amount of the extracellular fluid (ECF) volume. In CSWS, ECF loss will be seen along with urinary sodium loss, and hence patients will be in hypovolemic dehydrated state. Patients with SIADH may be either in euvolemic or hypervolemic condition. Management of CSWS patients ${ }^{5}$ is directed at correcting the sodium loss and ECF loss. Intravenous fluids, either isotonic or hypertonic saline, are used to correct intravascular volume and serum sodium depletion.

In intractable situations, aggressive management with hypertonic saline may be required several times a day to stabilize and improve sodium levels. Once euvolemia is achieved, the patient should be maintained in a positive salt balance and to prevent volume depletion by matching the urinary output with volume repletion. Close monitoring of serum sodium levels is important to assess the extent of correction and prevent overcorrection leading to osmotic demyelination syndrome. Fludrocortisone, a potent mineralocorticoid (in doses of $0.1-1 \mathrm{mg} / \mathrm{d}$ ), which causes reabsorption of sodium and water in the distal renal tubule, leading to expansion of the ECF, may be given in CSWS patients. Long-term therapy is not required, as CSWS is a transient condition that will usually resolve within 3 to 4 weeks, once the underlying cerebral pathology is corrected. A close coordination with the critical care team could be invaluable in the appropriate management of these patients.

\section{Conclusion}

As such, there is a reasonably good chance that most neurointerventionalists will encounter the diagnostic dilemma of persistent hyponatremia due to cerebrovascular diseases at some point in their career. Both SIADH and CSWS are potential etiologies, and their biochemical profiles have considerable overlapping findings. Biochemical and clinical assessment of the patient's extracellular volume status may help differentiate this condition. Accurate diagnosis is important, as the two conditions are treated differently.

\section{Contributions of Authors}

-AR-data collection, data analysis, data interpretation, manuscript preparation, and critical revision

- SKK-concept and design, data collection, data analysis, data interpretation, manuscript preparation, and critical revision

-SV-data analysis, data interpretation, and critical revision

-JER-data analysis, data interpretation, and critical revision

\section{Conflict of Interest}

The authors have no personal or financial conflict of interest to disclose.

\section{References}

1 Palmer BF. Hyponatraemia in a neurosurgical patient: syndrome of inappropriate antidiuretic hormone secretion versus cerebral salt wasting. Nephrol Dial Transplant 2000;15(02):262-268

2 Verbalis JG, Goldsmith SR, Greenberg A, et al. Diagnosis, evaluation, and treatment of hyponatremia: expert panel recommendations. Am J Med 2013;126(10, Suppl 1):S1-S42

3 Kao L, Al-Lawati Z, Vavao J, Steinberg GK, Katznelson L. Prevalence and clinical demographics of cerebral salt wasting in patients with aneurysmal subarachnoid hemorrhage. Pituitary 2009;12(04):347-351

4 See AP, Wu KC, Lai PM, Gross BA, Du R; AP. Risk factors for hyponatremia in aneurysmal subarachnoid hemorrhage. J Clin Neurosci 2016;32:115-118

5 Momi J, Tang CM, Abcar AC, Kujubu DA, Sim JJ. Hyponatremiawhat is cerebral salt wasting? Perm J 2010;14(02):62-65 